\title{
PRODUÇÃO DE BIOMASSA DE Pfaffia glomerata (Spreng.) PEDERSEN E Plantago major L. EM CULTIVO SOLTEIRO E CONSORCIADO
}

\author{
Pfaffia glomerata (Spreng.) Pedersen and Plantago major L. biomass \\ production in single culture and intercropped
}

\author{
Eduardo Xavier do Nascimento ${ }^{1}$, José Hortêncio Mota², Maria do Carmo Vieira ${ }^{3}$, Néstor Antonio Heredia Zárate ${ }^{3}$
}

\section{RESUMO}

O objetivo do trabalho foi avaliar a capacidade produtiva da Pfaffia glomerata (Spreng.) Pedersen e de Plantago major L., em cultivo solteiro e consorciado. Os tratamentos em estudo foram três e quatro fileiras de plantas de tansagem por canteiro, duas fileiras de fáfia espaçadas de $36 \mathrm{~cm}$ ou de $54 \mathrm{~cm}$, três fileiras de tansagem alternadas com duas fileiras de fáfia $(36 \mathrm{~cm})-\mathrm{T}_{3} \mathrm{~F}_{36}$ e quatro fileiras de tansagem alternadas com duas fileiras de fáfia $(54 \mathrm{~cm})-\mathrm{T}_{4} \mathrm{~F}_{54}$. Foi utilizado o delineamento blocos casualizados, com quatro repetições. Os espaçamentos entre plantas foram de $40 \mathrm{~cm}$ para fáfia e de $30 \mathrm{~cm}$ para tansagem. O tipo de cultivo e os espaçamentos entre fileiras não influenciaram significativamente a produção de massa fresca e seca da parte aérea e das raízes da fáfia. Na tansagem foi observado efeito significativo da forma de cultivo em todas as características avaliadas e do número de fileiras para o número de pendões. As alturas máximas das plantas de fáfia foram de $185 \mathrm{~cm}$ e $183 \mathrm{~cm}$, aos 172 e 164 dias após o transplante, sob cultivo solteiro, nos espaçamentos de $54 \mathrm{~cm}$ e $36 \mathrm{~cm}$, respectivamente. A razão de área equivalente (RAE), para o consórcio $\mathrm{T}_{3} \mathrm{~F}_{36}$ foi de 1,07 e para o $\mathrm{T}_{4} \mathrm{~F}_{54}$ foi de 1,50. No caso de recomendação agronômica, optar-se-ía pelo consórcio $\mathrm{T}_{4} \mathrm{~F}_{54}$.

Termos para indexação: Pfaffia glomerata, Plantago major, plantas medicinais, consórcio.

\begin{abstract}
This study aimed to evaluate the production of Pfaffia glomerata (Spreng.) Pedersen and Plantago major biomass in sole culture and intercropped. The treatments in study were three and four rows of plants of common plant for plat, two rows of $P$ faffia glomerata spaced of $36 \mathrm{~cm}$ or $54 \mathrm{~cm}$, three rows of common plant alternated with two rows of Pfaffia glomerata $(36 \mathrm{~cm})-\mathrm{T}_{3} \mathrm{~F} 3_{6}$ and four alternated rows of common plant with two rows of Pfaffia glomerata $(54 \mathrm{~cm})-\mathrm{T}_{4} \mathrm{~F}_{54}$. The experimental was desigm randomized blocks, with four replications. The spacing between plants was $40 \mathrm{~cm}$ for Pfaffia glomerata and $30 \mathrm{~cm}$ for common plant. The culture type and spacing between rows did not influence the fresh and dry roots and aerial part mass of the Pfaffia glomerata. In the common plant, it was observed significant effect of the form of culture in all the evaluated characteristics and number of rows for tassel. The maximum heights of the Pfaffia glomerata plants were $185 \mathrm{~cm}$ and $183 \mathrm{~cm}$ to the 172 and 164 days after transplanting, under single culture, for the $54 \mathrm{~cm}$ and $36 \mathrm{~cm}$ spacing, respectively. The area reason equivalent (RAE), for intercropping $\mathrm{T}_{3} \mathrm{~F}_{36}$ was 1.07 and for the $\mathrm{T}_{4} \mathrm{~F}_{54}$ was 1.50 . Therefore, the intercopping $\mathrm{T}_{4} \mathrm{~F}_{54}$, might be recomended.
\end{abstract}

Index terms: Pfaffia glomerata, Plantago major, medicinal plants, intercropped.

(Recebido em 27 de abril de 2006 e aprovado em 1 de dezembro de 2006)

\section{INTRODUÇÃO}

As plantas medicinais vêm adquirindo grande importância no mundo e no Brasil, com geração de emprego e renda. A indústria fitoterápica mundial movimenta cerca de US\$ 14 bilhões, ou seja, 5\% dos US\$ 280 bilhões que circulam no mercado global de medicamentos sintéticos (SOARES, 2002). No Brasil, estima-se que $82 \%$ da população brasileira utilize produtos a base de ervas, sendo que o setor fitoterápico movimenta anualmente $\mathrm{R} \$ 1$ bilhão em toda sua cadeia produtiva e emprega mais de $100 \mathrm{mil}$ pessoas (ABIFISA, 2006). Diante desses números deve-se otimizar a pesquisa de fitoterápicos no Brasil, como forma de proteger nossa biodiversidade, e também, desenvolver novos fármacos para a população.

Entre as plantas de interesse da flora medicinal brasileira pode-se citar a Pfaffia glomerata, conhecida como fáfia, ginseng do pantanal ou ginseng brasileiro, considerada como sucessora do ginseng verdadeiro (Panax ginseng C.A. Meyer). A fáfia tem grande potencial para consumo no mercado interno e, também, para exportação. De acordo com Ferreira (1998) e Montanari Júnior (2001), a fáfia produzida no Brasil é exportada principalmente, para o Japão e os Estados Unidos, sendo que o mercado brasileiro absorve quase toda a produção, não se comercializando mais por falta de matéria-prima.

\footnotetext{
Engenheiro Agrônomo - Universidade Federal da Grande Dourados/UFGD - Facudade de Ciências Agrárias/FCA - Cx. P. 533 - $79804-970$ Dourados, MS - Cx. P. 533 - 79.804-970 - exnascimento@bol.com.br

2Engenheiro Agrônomo, Doutor - Pesquisador DCR/CNPq - FUNDECT - Universidade Federal da Grande Dourados/UFGD - Facudade de Ciências Agrárias/FCA - Cx. P. 533 - 79804-970 - Dourados, MS - hortenciomota@terra.com.br

${ }^{3}$ Engenheiro Agrônomo, Doutor, Professor - Universidade Federal da Grande Dourados/UFGD - Faculdade de Ciências Agrárias/FCA - Cx. P. 533 79804-970 - Dourados, MS - vieiracm@terra.com.br; nahz@terra.com.br
} 
O potencial medicinal da fáfia deve-se ao fato de suas raízes apresentarem componentes que atuam na regeneração das células, na purificação do sangue, na inibição do crescimento de células cancerígenas, na regularização das funções hormonais e sexuais e como bioenergético (GAHAN, 1984), além de atuar como antidiabético (OLIVEIRA et al., 1980).

As plantas são perenes, ocorrem em orlas de matas, beiras de rios, capoeiras úmidas e campos rupestres. São subarbustivas ou arbustivas, com caules eretos ou semieretos, geralmente ocos, glabros ou levemente pilosos, com 0,5 a 2,5 m de altura. Possui raiz tuberosa e geralmente bifurcada, como do ginseng do Oriente, daí o nome ginseng brasileiro. As folhas são opostas, ovado-lanceoladas ou ovado-oblongas e pilosas; quando novas são largas e aquelas próximas das inflorescências são estreitas. A planta é típica de vegetação ciliar e de campos de inundação de rios, com ocorrência em todo o Brasil e países limítrofes; é freqüente no cerrado e matas do Estado de Mato Grosso do Sul (POTT \& POTT, 1994).

A fáfia é encontrada nas margens e ilhas do Rio Paraná, Paranapanema e Ivaí, entre o Estado de São Paulo, Mato Grosso do Sul e Paraná, onde vegeta naturalmente (OLIVEIRA et al., 1980). Encontra-se em risco de extinção, pela coleta intensiva de suas raízes nas poucas faixas de vegetação nativa ainda encontrada na região. Tal degradação poderia ser minimizada pelo processo de domesticação e cultivo dessas espécies, tornando-as, inclusive, disponíveis para uma parcela maior da população (MING \& CORREA JÚNIOR, 2001).

Outra espécie de interesse medicinal é a Plantago major conhecida como tanchagem, tansagem, transagem, tanchagem maior, plantagem ou língua de vaca. Espécie originária do continente europeu foi introduzida em diversas regiões do mundo, podendo ser encontrada em vários países da África, da Ásia e das Américas. No Brasil, as espécies, $P$. major e $P$. tomentosa Lam. foram encontradas vegetando espontaneamente, principalmente em áreas sob clima temperado, em associações com outras plantas, seja em jardins, gramados, pomares e/ ou beiras de estradas (BACCHI et al., 1984).

As partes das plantas de tansagem utilizadas como medicinais são as folhas, cujos principais constituintes do complexo bioquímico são flavonóides (alantoína), taninos, mucilagens, saponinas, ácidos orgânicos, sais de potássio, vitamina $\mathrm{C}$ e, nas sementes, antraquinonas (JORGE \& MARKMAN, 1994; KAWASLITY et al., 1994). Elas são utilizadas como expectorantes, antidiarréicas, cicatrizantes, adstringentes, emolientes, depurativas do sangue; além de utilizadas em inflamações bucofaríngeas, dérmicas, gastrintestinais e das vias urinárias; as sementes são laxativas. Em gargarejos, $P$. major e $P$. tomentosa são usadas contra inflamações da garganta, anginas e gengivas sangrentas. Além do valor medicinal, as plantas de Plantago major são consideradas hortaliças em potencial, por serem ricas em fósforo e cálcio e nas vitaminas A e C (FONT QUER, 1993; MARCHESAN et al., 1998; SAKER \& KAWASLITY, 1998; SILVA FILHO et al., 1994; SIQUEIRA \& PEREIRA, 1998).

As plantas de tansagem desenvolvem-se melhor em solos arenosos, ricos em matéria orgânica e com boa umidade (FONT QUER, 1993). Em relação aos tratos culturais, dessas e de outras plantas medicinais, especialmente no que se refere a espaçamentos/ populações de plantas e épocas de colheita, foram encontradas poucas citações. Os espaçamentos recomendados para tansagem variam de 0,20 a 0,30 m entre plantas x 0,50 m entre linhas, sendo as plantas colhidas entre dois a cinco meses após o plantio, antes da emissão das espigas (SILVA FILHO et al., 1994).

A ocorrência de consórcios agrícolas em diversas regiões indica que o número de interações ecológicas presentes nos agroecossistemas pode ser muito maior que o das consideradas nos modelos de agricultura convencional, em que a avaliação econômica, mais do que a biológica, determina os padrões de eficiência (HARDER et al., 2005). O consórcio de culturas é empregado, sobretudo pelos pequenos agricultores e pelos agricultores de subsistência, que contam com pequenas áreas para cultivos, mão-de-obra abundante para a área de que dispõem e pouco capital (VIEIRA, 1998). Uma das razões mais importantes para se cultivar duas ou mais culturas juntas é o aumento da produtividade por unidade de área e, em consequiência, induzir o aumento da renda bruta do produtor rural. Para tanto, deve-se considerar o arranjo espacial, densidade das plantas, data de maturação das culturas e arquitetura da planta. Isso, visando proporcionar maximização da cooperação e minimização da competição entre as espécies (SULLIVAN, 2001).

$\mathrm{Na}$ literatura consultada, não foram encontrados relatos sobre o consórcio da fáfia e a tansagem, espécies com características diferentes quanto à arquitetura, ciclo, exploração de solo e outros. Por isso, este trabalho teve como objetivo avaliar a capacidade produtiva da Pfaffia glomerata e de Plantago major, em cultivo solteiro e consorciado. 


\section{MATERIAL E MÉTODOS}

O experimento foi conduzido em área do Horto de Plantas Medicinais (HPM) da Universidade Federal da Grande Dourados (UFGD), em Dourados-MS, no período de agosto de 2004 a julho de 2005. O HPM situa-se em latitude de $22^{\circ} 11^{\prime} 43^{\prime \prime S}$, longitude de $54^{\circ} 56^{\prime} 08^{\prime \prime} \mathrm{W}$ e altitude de $458 \mathrm{~m}$.

O clima da região, segundo a classificação de Köppen, é Mesotérmico Úmido; do tipo Cwa, com temperaturas e precipitações médias anuais variando de $20^{\circ}$ a $24^{\circ} \mathrm{C}$ e de $1250 \mathrm{~mm}$ a $1500 \mathrm{~mm}$, respectivamente. $\mathrm{O}$ solo é do tipo Latossolo Vermelho distroférrico, com as seguintes características químicas, na profundidade de 0$20 \mathrm{~cm}$ : pH em água $=5,1 ; \mathrm{pH}$ em CaCl: 4,5; M.O. $=31,5$ $\mathrm{g} \mathrm{dm}^{-3} ; \mathrm{P}=14 \mathrm{mg} \mathrm{dm}^{-3}$; e 0,$9 ; 2,8 ; 29,6 ; 16,3 ; 89,0 ; 46,8$ e $135,8 \mathrm{mmol}_{\mathrm{c}} \mathrm{dm}^{-3}$ de $\mathrm{K}, \mathrm{Al}, \mathrm{Ca}$ e $\mathrm{Mg}, \mathrm{H}+\mathrm{Al}, \mathrm{SB}$ e CTC, respectivamente. Os resultados das análises físicas foram: 760,0; 90,9; 89,2 e 60,2 $\mathrm{g} \mathrm{kg}^{-1}$ de argila, silte, areia grossa e areia fina, respectivamente; densidade do solo $=1,12 \mathrm{e}$ densidade de partículas $=2,08$.

Foram considerados dois tratamentos consorciados: três fileiras de tansagem com duas fileiras de fáfia espaçadas de $36 \mathrm{~cm}$, e, quatro fileiras de tansagem com duas fileiras de fáfia espaçadas de $54 \mathrm{~cm}$. Já os tratamentos solteiros foram: fáfia cultivada em duas fileiras (36 cm e $54 \mathrm{~cm}$ de espaçamento) e tansagem cultivada sob três (33 cm de espaçamento) e quatro fileiras $(25 \mathrm{~cm}$ de espaçamento). Foi utilizado o delineamento blocos casualizados, com quatro repetições. Os espaçamentos entre plantas foram de $40 \mathrm{~cm}$ para fáfia e de $30 \mathrm{~cm}$ para tansagem. A área total de cada parcela foi de 4,5 $\mathrm{m}^{2}(3,0 \mathrm{~m}$ de comprimento e 1,5 $\mathrm{m}$ de largura) e área útil de 3,0 $\mathrm{m}^{2}(3,0 \mathrm{~m}$ de comprimento e 1,0 $\mathrm{m}$ de largura).
A propagação da fáfia e da tansagem foi por semeadura indireta. As sementes foram colhidas em plantas remanescentes em área do HPM, peneiradas e posteriormente semeadas em bandejas de plástico com substrato comercial. Aos 30 dias após a semeadura, as plântulas de cada espécie foram repicadas para bandejas de poliestireno de 128 células, onde permaneceram por mais 55 dias, quando, então, foi realizado o transplante ao local definitivo.

Durante o ciclo de cultivo da fáfia foram feitas nove medições das alturas de todas as plantas de cada parcela, a partir de 30 dias do transplante e a intervalos regulares de 30 dias. A colheita, feita aos 330 dias, consistiu no arranquio das plantas inteiras, quando avaliaram-se as massas frescas e secas das partes aéreas e das raízes. As plantas de tansagem foram colhidas logo que as primeiras espigas foram emitidas, o que ocorreu aos 119 dias após a semeadura, sendo as plantas cortadas rente ao solo e avaliadas a área foliar, as massas fresca e seca da parte aérea e os números de folhas e de pendões. As médias foram testadas pelo teste $\mathrm{F}$, a $5 \%$ de probabilidades.

O consórcio foi avaliado utilizando a expressão da razão de área equivalente (RAE) proposto por Caetano et al. (1999), a saber: $\mathrm{RAE}=\mathrm{Fc} \cdot \mathrm{Fs}^{-1}+\mathrm{Tc} \cdot \mathrm{Ts}^{-1}$, onde, respectivamente, $\mathrm{Fc}$ e $\mathrm{Tc}=$ produções de massa fresca da fáfia e da tansagem em consorciação e Fs e Fs = produções de massa fresca da fáfia e da tansagem em cultivo solteiro.

\section{RESULTADOS E DISCUSSÃO}

Os resultados obtidos mostraram que o tipo de cultivo e os espaçamentos entre fileiras não influenciaram significativamente a produção de massa fresca e seca da parte aérea e das raízes da fáfia (Tabela 1).

TABELA 1 - Produção de massas fresca e seca da parte aérea e das raízes de fáfia, em cultivo solteiro e em consórcio com tansagem, sob duas ou três fileiras no canteiro. UFGD, Dourados, 2005.

\begin{tabular}{ccccc}
\hline \multirow{2}{*}{ Tratamentos } & \multicolumn{2}{c}{ Massa de parte aérea $\left(\mathbf{k g ~ h a}^{\mathbf{- 1}}\right)$} & \multicolumn{2}{c}{ Massa de raízes $\left(\mathbf{k g ~ h a} \mathbf{~}^{-\mathbf{1}}\right)$} \\
\cline { 2 - 5 } Cultivo & Fresca & Seca & Fresca & Seca \\
\hline Solteiro & & & & $3038,7 \mathrm{a}$ \\
Consórcio & $21193,8 \mathrm{a}$ & $6041,0 \mathrm{a}$ & $11581,9 \mathrm{a}$ & $2273,3 \mathrm{a}$ \\
\hline Fileiras & $17286,0 \mathrm{a}$ & $5176,2 \mathrm{a}$ & $8738,3 \mathrm{a}$ & $2348,8 \mathrm{a}$ \\
\hline Duas $(54 \mathrm{~cm})$ & $17132,7 \mathrm{a}$ & & $8783,5 \mathrm{a}$ & $2963,3 \mathrm{a}$ \\
Duas $(36 \mathrm{~cm})$ & $21347,1 \mathrm{a}$ & $5199,1 \mathrm{a}$ & $11536,7 \mathrm{a}$ & 30,10 \\
\hline C.V. $(\%)$ & 32,34 & $6018,1 \mathrm{a}$ & 27,18 & 32,54 \\
\hline
\end{tabular}

Médias seguidas pelas mesmas letras, nas colunas, não diferem, pelo teste $\mathrm{F}$, a $5 \%$ de probabilidade.

Ciênc. agrotec., Lavras, v. 31, n. 3, p. 724-730, maio/jun., 2007 
Apesar de não haver diferença estatística entre os sistemas de cultivo, houve diferenças de 18,4\%, 14,3\%, $24,5 \% 25,2 \%$ a mais nas produções de massas fresca e seca de parte aérea e raízes, respectivamente, no cultivo solteiro de fáfia. Já para as fileiras, houve diferenças de $19,7 \%, 15,6 \%, 31,3 \%$ e $20,7 \%$ a mais nas produções de massas fresca e seca de parte aérea e raízes, respectivamente, no cultivo sob $36 \mathrm{~cm}$ entre fileiras de fáfia. Santos (1998) relata que as espécies podem apresentar mecanismos de compensação da produtividade que podem ocorrer em função de modificações das populações delas nas associações e nos arranjos espaciais, ou mesmo em função do sincronismo de plantio e do desenvolvimento temporal das espécies.

As produções de massa fresca e seca da fáfia obtidas neste experimento, aos 300 dias após o transplante, foram superiores às das plantas de idade semelhante relatadas por Montanari Júnior et al. (1997). Estes, estudando Pffafia glomerata, constataram que a produtividade de matéria fresca de raiz foi de 7,2;12,7 e $15,8 \mathrm{t} \mathrm{ha}^{-1}$ e de matéria seca 2,2; 3,8 e 4,8 $\mathrm{t} \mathrm{ha}^{-1}$ aos $12,24 \mathrm{e}$ 36 meses, respectivamente.

$\mathrm{Na}$ tansagem foi observado efeito significativo da forma de cultivo em todas as características avaliadas e efeito do número de fileiras de plantas para o número de pendões. (Tabela 2).

As maiores produções de pendões e de folhas foram das plantas cultivadas solteiras mostrando que, no consórcio, se chegou à pressão populacional. Ele diminuiu as capacidades produtivas totais das plantas da fáfia devido à competição por fatores de crescimento, tais como luz, nutrientes e água, o que poderia resultar em decréscimo da produção (MARSCHNER, 1995). O número de pendões foi maior no tratamento quatro fileiras, mas as massas frescas e secas foram, estatisticamente, semelhantes mostrando que, provavelmente, houve modificações na plasticidade fisiológica da planta para adaptar-se a essas condições do ambiente. Entretanto, observaram-se diferenças de $5,5 \%, 12,9 \%, 23,7 \%, 19,8 \%$ e $19,9 \%$ a mais nas produções de número, massas fresca e seca de pendões e folhas no cultivo em quatro fileiras de tansagem. Já para a área foliar foi $8,1 \%$ superior no cultivo com três fileiras.

A altura das plantas de fáfia apresentou crescimento quadrático, independente da forma de cultivo (Figura 1). As alturas máximas foram de $185 \mathrm{~cm}$ e $183 \mathrm{~cm}$, aos 172 e 164 dias após o transplante, sob cultivo solteiro, nos espaçamentos de $54 \mathrm{~cm}$ e $36 \mathrm{~cm}$, respectivamente. As alturas mínimas foram de $177 \mathrm{~cm}$ e $178 \mathrm{~cm}$ nos consórcios duas fileiras de fáfia a $54 \mathrm{~cm}$ com quatro fileiras de tansagem e duas fileiras de fáfia a $36 \mathrm{~cm}$ com três fileiras de tansagem, respectivamente. Esses resultados indicam que, as plantas solteiras tiveram melhor adaptabilidade, normalmente sendo esta relacionada com a manutenção da eficiência na absorção ou no uso da água, dos nutrientes e do $\mathrm{CO}_{2}$ (LARCHER, 2000).

A razão de área equivalente (RAE), para o consórcio três fileiras de tansagem alternadas com duas fileiras de fáfia $(36 \mathrm{~cm})\left(\mathrm{T}_{3} \mathrm{~F}_{36}\right)$, considerando as produtividades de massa fresca das folhas de tansagem e das raízes de fáfia, foi de $1,07\left[\operatorname{RAE~T} \mathrm{F}_{36}=(6.601,68,10.965,40)+(2987,85\right.$ $, 6 \cdot 303,18)=0,60+0,47=1,07]$. A RAE para o consórcio

TABELA 2 - Número e massas fresca e seca de pendões e área foliar, número e massa fresca e seca de folhas de tansagem, em cultivo solteiro e em consórcio com fáfia, sob três e quatro fileiras por canteiro. UFGD, Dourados, 2005.

\begin{tabular}{|c|c|c|c|c|c|c|c|}
\hline \multirow{3}{*}{ Tratamento } & \multicolumn{3}{|c|}{ Pendões } & \multicolumn{4}{|c|}{ Folhas } \\
\hline & \multirow{2}{*}{ Número } & \multicolumn{2}{|c|}{$\operatorname{Massa}\left(\mathrm{kg} \mathrm{ha}^{-1}\right)$} & \multirow{2}{*}{ Número } & \multicolumn{2}{|c|}{$\operatorname{Massa}\left(\mathrm{kg} \mathrm{ha}^{-1}\right)$} & \multirow{2}{*}{$\begin{array}{l}\text { Área foliar } \\
\quad\left(\mathrm{cm}^{2}\right)\end{array}$} \\
\hline & & Fresca & Seca & & Fresca & Seca & \\
\hline \multicolumn{8}{|l|}{ Cultivo } \\
\hline Solteiro & $728 \mathrm{a}$ & $1725,2 \mathrm{a}$ & $318,0 \mathrm{a}$ & $1486,1 \mathrm{a}$ & $6784,3 \mathrm{a}$ & $1339,2 \mathrm{a}$ & $1799,22 \mathrm{a}$ \\
\hline Consórcio & $439 \mathrm{~b}$ & $952,0 \mathrm{~b}$ & $174,8 \mathrm{~b}$ & $1086,1 \mathrm{~b}$ & $3424,6 \mathrm{~b}$ & $920,8 \mathrm{~b}$ & $1095,80 \mathrm{~b}$ \\
\hline \multicolumn{8}{|l|}{ Fileiras } \\
\hline Três & $500 \mathrm{a}$ & $1303,0 \mathrm{a}$ & $231,5 \mathrm{a}$ & $1150,0 \mathrm{a}$ & $4645,5 \mathrm{a}$ & $1027,7 \mathrm{a}$ & $1506,28 \mathrm{a}$ \\
\hline Quatro & $667 \mathrm{~b}$ & $1374,2 \mathrm{a}$ & $261,3 \mathrm{a}$ & $1422,2 \mathrm{a}$ & $5563,3 \mathrm{a}$ & $1232,2 \mathrm{a}$ & $1385,74 \mathrm{a}$ \\
\hline C.V. $(\%)$ & 20,37 & 44,18 & 40,22 & 19,41 & 18,78 & 17,54 & 16,66 \\
\hline
\end{tabular}

Médias seguidas pelas mesmas letras, nas colunas, não diferem, pelo teste $\mathrm{F}$, a $5 \%$ de probabilidade. 


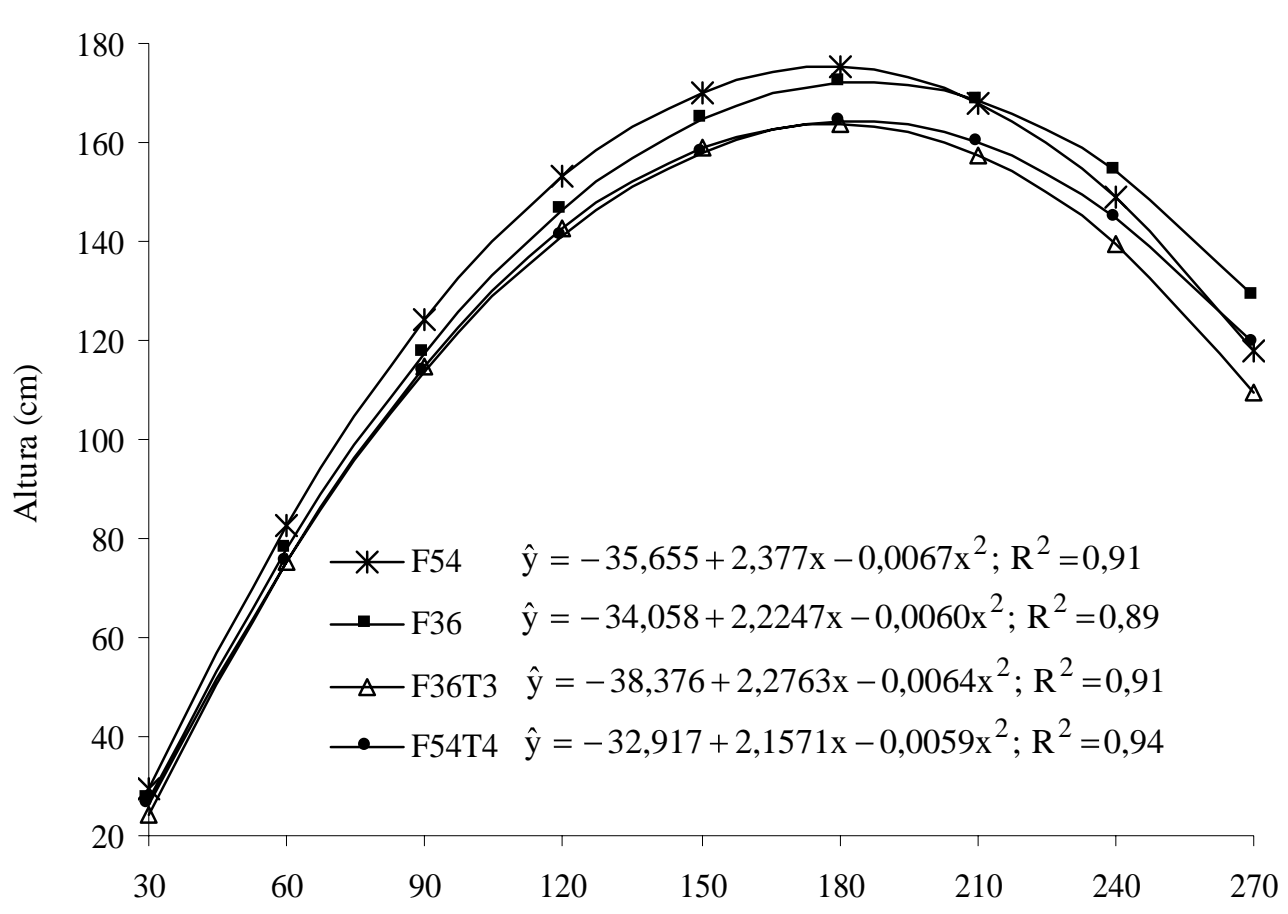

Dias após o transplante

FIGURA 1 - Altura das plantas de fáfia avaliadas em cultivo solteiro e consorciado com tansagem, em diferentes espaçamentos. Em que: F54 = duas fileiras de fáfia a $54 \mathrm{~cm}$; F36 = duas fileiras de fáfia a $36 \mathrm{~cm}$; F36T3 = duas fileiras de fáfia a $36 \mathrm{~cm}$ com três fileiras de tansagem; F54T4 = duas fileiras de fáfia a $54 \mathrm{~cm}$ com quatro fileiras de tansagem. UFGD, Dourados, 2005.

quatro fileiras de tansagem alternadas com duas fileiras de fáfia $(54 \mathrm{~cm})\left(\mathrm{T}_{4} \mathrm{~F}_{54}\right)$ foi de 1,50 [RAE $\mathrm{T}_{4} \mathrm{~F}_{54}=(10.875,00$, $12.198,33)+(3.861,35,6.303,18)=0,89+0,61=1,50]$. Pelo fato de os valores das RAEs terem sido superiores a 1,0 , conclui-se que os consórcios entre a tansagem e a fáfia foram efetivos. No caso de recomendação agronômica optarse-ía pelo consórcio quatro fileiras de tansagem alternadas com duas fileiras de fáfia, com espaçamento de $54 \mathrm{~cm}$.

\section{CONCLUSÕES}

Não houve influência significativa no tipo de cultivo e nos espaçamentos entre fileiras para a produção de massa fresca e seca da parte aérea e das raízes da fáfia.

As alturas máximas das plantas de fáfia, sob cultivo solteiro, foram de $185 \mathrm{~cm}$ e $183 \mathrm{~cm}$, aos 172 e 164 dias após o transplante, nos espaçamentos de $54 \mathrm{~cm}$ e $36 \mathrm{~cm}$, respectivamente.

Para a tansagem, o cultivo solteiro propiciou maiores rendimentos de número, massas fresca e seca de pendões e folhas.
O cultivo de tansagem em três e quatro fileiras propiciou produções estatisticamente semelhantes, com exceção do número de pendões que foi superior no cultivo em quatro fileiras.

A razão de área equivalente para o consórcio $\mathrm{T}_{3} \mathrm{~F}_{36}$ foi de 1,07 e para $\mathrm{o}_{4} \mathrm{~F}_{54}$ foi de 1,50, sendo recomendado $\mathrm{o}$ consórcio $\mathrm{T}_{4} \mathrm{~F}_{54}$.

\section{AGRADECIMENTOS}

Ao CNPq, pelas bolsas concedidas e à FUNDECT, pelo apoio financeiro.

\section{REFERÊNCIAS BIBLIOGRÁFICAS}

ASSOCIAÇÃO BRASILEIRA DAS EMPRESAS DO SETOR FITOTERÁPICO. Uma legislação justa para os produtos de origem natural. Disponível em: <http:// www.abifisa.org.br/introducao.asp>. Acesso em: 24 fev. 2006. 
BACCHI, O.; LEITÃO FILHO, H. de F.; ARANHA, C. Plantas invasoras de culturas. Campinas: Instituto Campineiro de Ensino Agrícola, 1984. 906 p.

CAETANO, L. C. S.; FERREIRA, J. M.; ARAÚJO, M. de. Produtividade da alface e cenoura em sistema de consorciação. Horticultura Brasileira, Brasília, v. 17, n. 2, p. 143-146, 1999.

FERREIRA, S. H. Medicamentos a partir de plantas medicinais no Brasil. São Paulo: Academia Brasileira de Ciências, 1998. 141 p. Disponível em: <http:// www.abc.org.br/arquivos/medicamentos.pdf $>$. Acesso em: 30 set. 2005.

FONT QUER, P. Plantas medicinales: el dioscórides renovado. Madri: Editorial Labor, 1993. 3 v.

GAHAN, P. B. Plant histochemistry and cytochemistry: an introduction. London: Academic, 1984. 301 p.

HARDER, W. C.; ZÁRATE, N. A. H.; VIEIRA, M. C. Produção e renda bruta de rúcula (Eruca sativa Mill.) 'Cultivada' e de almeirão (Cichorium intybus L.) 'Amarelo', em cultivo solteiro e consorciado. Ciência e Agrotecnologia, Lavras, v. 29, n. 4, p. 775-785, 2005.

JORGE, L. F.; MARKMAN, B. E. O. Caracterização histológica e química (cromatografia em camada delgada) de Plantago tomentosa Lam. (Tanchagem). Revista Brasileira de Farmácia, São Paulo, v. 75, n. 1, p. 10-12, 1994.

KAWASLITY, A. A.; GAMAL-EL-DIN, E.; ABDALLA, M. F.; SALEH, A. M. Flavonoides of Plantago species in Egipt. Biochemical Systematics and Ecology, [S.1.], v. 22, n. 7, p. 729-733, 1994.

LARCHER, W. Ecofisiologia vegetal. São Carlos: RiMa Artes e Textos, 2000. 531 p.

MARCHESAN, M.; PAPER, D. H.; HOSE, S.; FRANZ, G. Investigation of the antiinflamatory activity of liquid extracts of Plantago lanceolata L. Phytotherapy Research, [S.1.], v. 12, p. 33-34, 1998.

MARSCHNER, H. Mineral nutrition of higher plants. London: Academic, 1995. 902 p.
MING, L. C.; CORREA JÚNIOR, C. Coleta de fáfia [Pfaffia glomerata (Spreng.) Pedersen] na região noroeste do estado do Paraná - Brasil. Acta Horticulturae, The Hague, n. 576, p. 29-32, 2001.

MONTANARI JUNIOR, I. Aspectos do cultivo de plantas medicinais nativas. Agroecologia Hoje, [S.1.], v. 7, 2001.

MONTANARI JÚNIOR, I.; MAGALHÃES, P.; QUEIROGA, C. L. Influence of plantation density and cultivation cycle in rot productivity and tenors of becdisone in Pffafia glomerata (Spreng) Pederson. In: WORLD CONGRESS ON MEDICINALAND AROMATIC PLANTS FOR HUMAN WELFARE, 2., 1997, Mendoza. Abstracts... Mendoza: [s.n.], 1997.

OLIVEIRA, F.; AKISUE, G.; AKISUE, M. K. Contribuição para o estudo farmacognóstico do Ginseng brasileiro, Pfaffia paniculata (Martius) Kuntze. Anais de Farmácia e Química, São Paulo, v. 20, p. 261-277, 1980.

POTT, A.; POTT, V. S. Plantas do pantanal. Corumbá: Embrapa-SPI, 1994. 320 p.

SAKER, M. M.; KAWASLITY, S. A. Tissue culture and flavonoid content of Nepeta and Plantago species endemic in Egypt. Fitoterapia, [S.1.], v. 69, n. 4, p. 358-364, 1998.

SANTOS, R. H. S. Interações interespecíficas em consórcio de olerícolas. 1998. 129 f. Tese (Doutorado em Fitotecnia) - Universidade Federal de Viçosa, Viçosa, 1998.

SILVA FILHO, P. V. da; LACA-BUENDIA, J. P.; OLIVEIRA, L. M. da S.; REZENDE, W. M. Ciclo biológico de duas espécies do gênero Plantago L. ocorrentes no Estado de Minas Gerais. Daphne, Belo Horizonte, v. 4, n. 1, p. 39-45, 1994.

SIQUEIRA, J. M.; PEREIRA, N. F. Plantago tomentosa como planta medicinal em Mato Grosso do Sul. In: SIMPÓSIO DE PLANTAS MEDICINAIS DO BRASIL, 15., 1998, Águas de Lindóia. Resumos... Águas de Lindóia: SBPM, 1998.

SOARES, A. C. Se bem não fizer, mal também não fará. Revista Eletrônica de Ciências, São Carlos, n. 12, 2002. Disponível em: <http://www.cdcc.sc.usp.br/ciencia/artigos/ art_12/medicamento.html>. Acesso em: 30 set. 2005. 
SULLIVAN, P. Intercropping principles and production pratices. 2001. Disponível em: <http://www.attra.org/ attra-pub/intercrop.html\#abstract $>$. Acesso em: 3 set. 2003.
VIEIRA, C. Cultivos consorciados. In: VIEIRA, C.; PAULA JÚNIOR, T. J.; BORÉM, A. (Eds.). Feijão: aspectos gerais e cultura no Estado de Minas Gerais. Viçosa: UFV, 1998. p. 523-558.

Ciênc. agrotec., Lavras, v. 31, n. 3, p. 724-730, maio/jun., 2007 